\title{
Extension of high-order harmonic generation cutoff via coherent control of intense few-cycle chirped laser pulses
}

\author{
Juan J. Carrera and Shih-I Chu \\ Chemistry Department, University of Kansas and Kansas Center for Advanced Scientific Computing, Lawrence, Kansas 66045, USA
}

(Received 18 September 2006; published 16 March 2007)

\begin{abstract}
We present an ab initio quantum investigation of the high-order harmonic generation (HHG) cutoff extension using intense few-cycle chirped laser pulses. For a few-cycle chirped driving laser pulse, it is shown that significant cutoff extension can be achieved through the optimization of the chirping rate parameters. The HHG power spectrum is calculated by solving accurately and efficiently the time-dependent Schrödinger equation by means of the time-dependent generalized pseudospectral method. The time-frequency characteristics of the HHG power spectrum are analyzed in detail by means of the wavelet transform of the time-dependent induced dipole acceleration. In addition, we perform classical trajectory simulation of the strong-field electron dynamics and electron return map. It is found that the quantum and classical results provide complementary and consistent information regarding the underlying mechanisms responsible for the substantial extension of the cutoff region.
\end{abstract}

DOI: 10.1103/PhysRevA.75.033807

Recent developments in attosecond metrology have allowed the production of laser pulses with reproducible temporal evolution of the electric field by using phase-stabilized optical frequency combs [1,2]. Attosecond laser pulses can be produced by means of the high-order harmonic generation (HHG) process in rare gases [3-5]. Coherent control techniques can be applied to the study of the interaction between matter and laser fields to obtain a desirable outcome by manipulating the temporal shape of the interacting laser field with high precision [6]. It has also been shown theoretically and experimentally that the enhancement of selective harmonics can be accomplished by adjusting the pulse shape of the excitation laser pulse via an evolutionary algorithm optimization scheme $[7,8]$. Similar HHG enhancement can be achieved by coherently controlling the dynamics of the electron wave packet via chirping the femtosecond laser pulse [9]. Indeed, there has been an increasing interest recently in the use of frequency- and amplitude-chirped pulses to perform quantum control of physical and chemical processes. For instance, chirped pulses have been applied to the control of population transfer in Rydberg atoms [10], and in the measurement of attosecond electronic dynamics [11].

In this paper, we present a detailed ab initio quantum investigation of the HHG cutoff extension by means of the optimization of the chirping parameters of intense few-cycle driving laser pulses. The time-dependent Schrödinger equation (TDSE) is solved accurately and efficiently by means of the time-dependent generalized pseudospectral (TDGPS) technique [12]. The time-frequency characteristics of the emitted pulses are analyzed by means of the wavelet transform of the induced dipole acceleration [13]. Our results show that a significant extension in the harmonic generation cutoff can be realized by the use of optimized chirped laser pulses. Since the extended cutoff position is now located at higher frequency, the time duration of the attosecond pulse produced is also reduced.

The HHG and attosecond xuv pulse generation [14] can be studied by solving the following TDSE (in atomic units):
PACS number(s): 42.65.Ky, 42.65.Re, 32.80.Rm

$$
i \frac{\partial}{\partial t} \psi(\mathbf{r}, t)=\hat{H} \psi(\mathbf{r}, t)=\left[\hat{H}_{0}+\hat{V}(\mathbf{r}, t)\right] \psi(\mathbf{r}, t) .
$$

Here, $\hat{H}_{0}$ is the field-free Hamiltonian of atomic hydrogen, and $\hat{V}(\mathbf{r}, t)$ is the time-dependent atom-field interaction. For a linearly polarized laser field $(\mathbf{F} \| \hat{z}), \hat{V}(\mathbf{r}, t)$ can be expressed in the length form [15] as

$$
\hat{V}(\mathbf{r}, t)=-\mathbf{F} \cdot \mathbf{r} E(t)=-F z f(t) \cos [\omega t+\delta(t)],
$$

where $f(t)$ is the laser field envelope, $\omega$ the laser frequency, and $F$ the peak laser field amplitude. The time profile of the carrier envelope phase (CEP), $\delta(t)$, considered in this work, has the following time-varying hyperbolic tangent form:

$$
\delta(t)=-\beta \tanh \left(\frac{t-t_{0}}{\tau}\right) .
$$

The chirp form is controlled by adjusting the two parameters $\beta$ (rad) (for controlling the frequency sweeping range), and $\tau$ (a.u.) (for controlling the steepness of the chirping function). $t_{0}$ is set at the middle of the sweep. The instantaneous frequency of the pulse is then of the form

$$
\omega(t)=\omega+d \delta(t) / d t=\omega-\frac{\beta}{\tau} \frac{1}{\cosh ^{2}\left(\frac{t-t_{0}}{\tau}\right)} .
$$

When $\beta=0$, the CEP is 0 , and the pulse is chirp-free. Due to the recent advancement of comb laser technology $[2,16]$, it is highly likely that such a time-varying CEP can be achieved in the near future. Note that other forms of frequency-chirped pulses have already been employed experimentally, for example, in driving multiphoton transitions between Rydberg states $[10,17]$.

Equation (1) is solved accurately and efficiently by means of the time-dependent generalized pseudo spectral (TDGPS) method [12]. The TDGPS technique has been shown to be considerably more accurate and computationally more effi- 

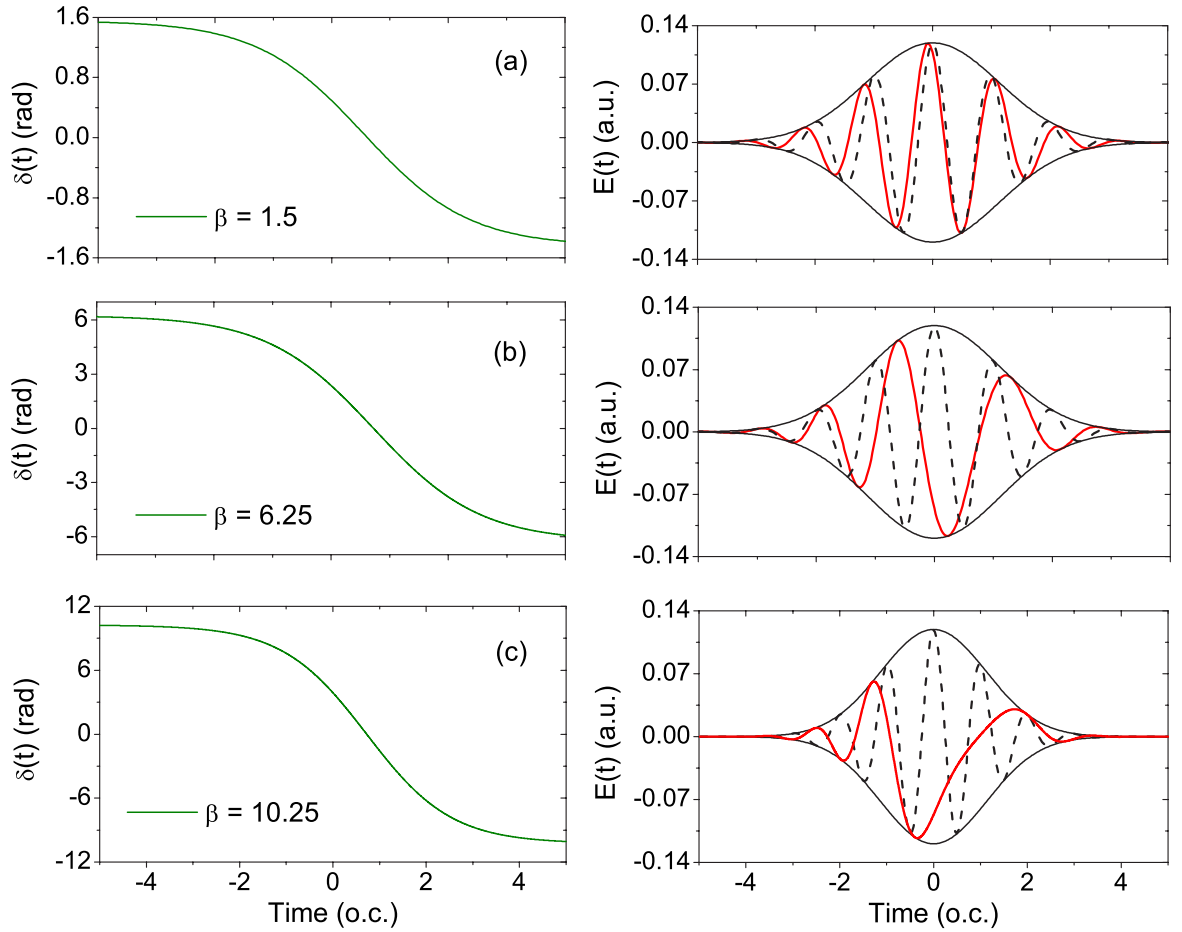

FIG. 1. (Color online) Effects of the chirped frequency on the laser field. (a)-(c) in the left column indicate the time-dependent CEP $\delta(t)$ modeled by a hyperbolic tangent function. The corresponding individual laser pulse is shown (solid line) in the right column. The dotted lines indicate the corresponding chirp-free $(\beta=0)$ laser pulses. cient than the conventional time-dependent propagation techniques using equal-spacing grid discretization [18]. It has been successfully applied to the study of field-induced Rydberg-atom high-resolution spectroscopy [19], and strongfield HHG processes of atomic $[12,13,20]$ and molecular $[21,22]$ systems. Recently, the TDGPS method has been further extended to the fully ab initio investigation of the HHG of helium atoms, involving the accurate solution of a sixdimensional TDSE [23]. The numerical scheme of the TDGPS method consists of two essential steps. (a) The spatial coordinates are optimally discretized in a nonuniform spatial grid by means of the generalized pseudospectral technique [24]. This discretization is characterized by denser grids near the nuclear origin and sparser grids for larger distances. (b) A second-order split-operator technique in the energy representation, which allows the explicit elimination of undesirable fast-oscillating high-energy components, is used for the efficient time propagation of the wave function [12],

$$
\begin{aligned}
\psi(\mathbf{r}, t+\Delta t) \simeq & \exp \left(-i \hat{H}_{0} \Delta t / 2\right) \exp [-i \hat{V}(r, \theta, t+\Delta t / 2) \Delta t] \\
& \times \exp \left(-i \hat{H}_{0} \Delta t / 2\right) \psi(\mathbf{r}, t)+O\left(\Delta t^{3}\right) .
\end{aligned}
$$

The unitarity of the wave function is automatically preserved by Eq. (5) and the norm of the field-free wave function is preserved to at least ten digits of accuracy during the TDGPS time propagation of the wave function. Having determined the time-dependent wave function $\psi(\mathbf{r}, t)$, we can then calculate the time-dependent induced dipole acceleration as [12]

$$
d_{A}(t)=\left\langle\psi(\mathbf{r}, t)\left|-\frac{z}{r^{3}}+F f(t) \cos [\omega t+\delta(t)]\right| \psi(\mathbf{r}, t)\right\rangle,
$$

from which the HHG power spectrum can be determined.

We performed the calculations with few-cycle chirped pulses corresponding to laser parameters of 5 fs full width at half maximum Gaussian laser pulse with peak intensity $I=5 \times 10^{14} \mathrm{~W} / \mathrm{cm}^{2}$ and wavelength $\lambda=800 \mathrm{~nm}$. First of all, we present the effects of chirped frequency on the laser pulse. The left column of Figs. 1(a)-1(c) shows the hyperbolic tangent form of the time-varying CEP $\delta(t)$ achieved by fixing $\tau=200$ a.u., while varying the $\beta$ parameter. The corresponding laser pulse is shown in the right column. Reshaping the laser pulse by chirping the frequency of the laser field has a direct consequence of breaking the oscillatory periodicity of the laser field. On the other hand, the amplitude of the Gaussian envelope laser pulse remains invariant to the chirped frequency. Second, we investigate the HHG power spectra under the influence of a chirped laser pulse with increasing $\beta$ values as shown in Figs. 2(a)-2(d). One notable feature common to the presented HHG power spectra is the appearance of a quasicontinuum region near the cutoff regime displaying almost structureless patterns. More importantly, the cutoff position for the $\beta=6.25$ case [Fig. 2(c)] is markedly extended, compared with the $\beta=0$ (chirp-free) case [Fig. 2(a)]. Indeed, a cutoff extension of at least 150 harmonic orders is observed between the cases of $\beta=6.25$ and $\beta=0$. If the $\beta$ parameter is further increased, as is seen in Fig. 2(d) for $\beta=10.25$, the cutoff extension is not as far reaching as in the case of Fig. 2(c). Thus the optimal cutoff extension can be achieved by adjusting $\beta$. Finally, when $\tau$ is varied while fixing $\beta$, the HHG cutoff position does not change considerably. Consequently, the extension of the HHG cutoff position is much more dependent on $\beta$ than on $\tau$. On the other hand, if $\beta<0$, then the cutoff position will be shortened and will emerge at considerably lower frequency than that of $\beta>0$.

We note that the strong-field approximation (SFA) is commonly used in the investigation of HHG processes, particularly for the case of longer laser pulse excitation [25]. However, the SFA is based on several approximations, including 

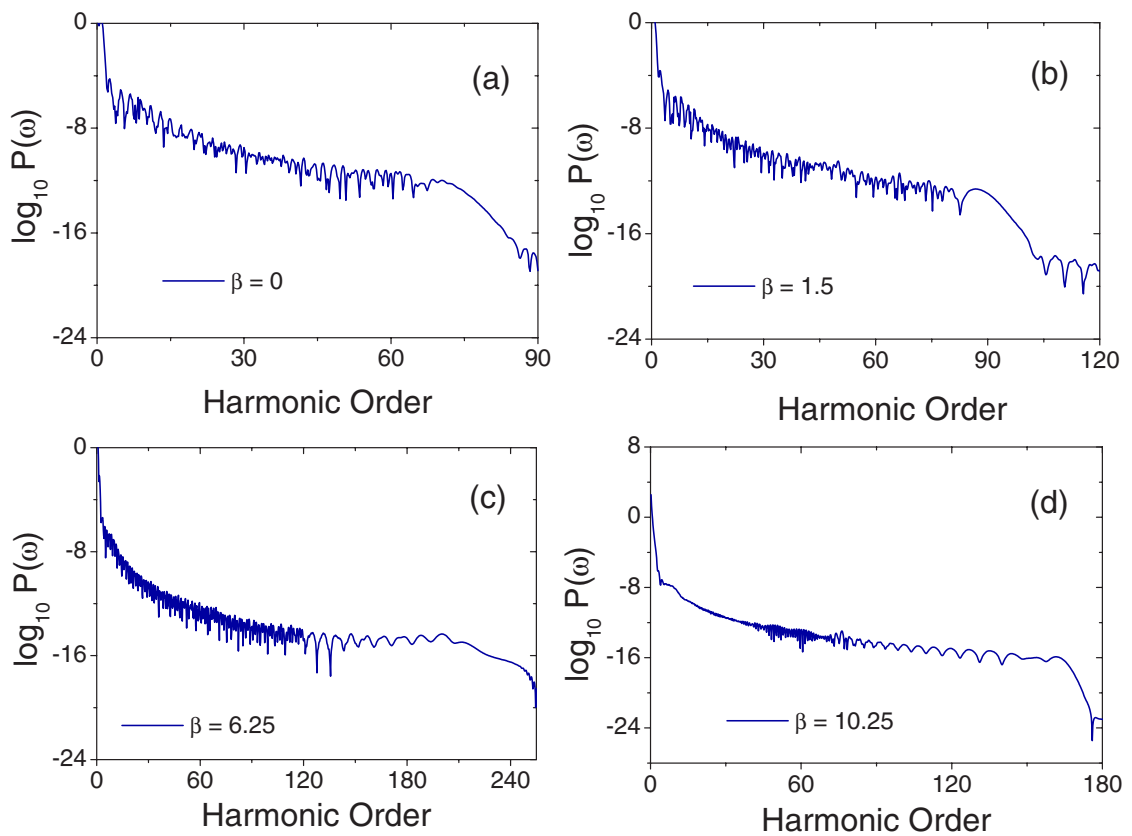

FIG. 2. (Color online) Effects of the driving chirped laser pulse on the HHG power spectra for $\mathrm{H}$ atoms. (a)-(d) indicate the power spectra generated by various sweeping parameters $\beta$ (in rad) for (a) 0 , (b) 1.5 , (c) 6.25 , and (c) 10.25 , respectively. (a) negligible ionization of the ground state, (b) dropping the Coulomb potential and treating only the ground and the (free-particle) continuum states, and (c) limitation to the weak or medium strong monochromatic laser fields. Due to the large extent of depletion of the ground state's population (not shown) caused by the ultrashort chirped laser pulse, and the lack of field periodicity of the laser, the SFA can only provide qualitative results in this case. Furthermore, the continuum states need to be treated more accurately.

To facilitate the exploration of the underlying mechanism responsible for the cutoff extension driven by a few-cycle chirped laser pulse, we perform a classical simulation of the electron dynamics. According to the semiclassical model [26], the HHG process consists of the following three steps. First, the electron tunnels through the barrier formed by the atomic Coulomb potential and the laser field. It then oscillates quasifreely driven by the Lorenz force and acquires kinetic energy from the laser field. Lastly, after the laser reverses its direction, the returning electron will emit harmonic photons by radiative recombination with the parent ion core. The photon energy of the highest harmonic of the spectrum is then determined approximately by the maximum kinetic energy gained by the electron as $I_{p}+3.17 U_{p}$, where $U_{p}$ is the quiver energy of free electrons in an oscillating field and $I_{p}$ the ionization potential.

Chirping the laser pulse will modify the trajectory of the electron wave packet. Figures 3(a) and 3(b) show the chirpfree and chirped driving laser fields, respectively. The parameters used for the chirped laser pulse are $\beta=6.25 \mathrm{rad}$ and $\tau=200$ a.u. The tunneling electron wave packet will be launched when the laser field reaches a local maximum. In the case of the chirp-free laser pulse, there are three major local maximum points $A, B$, and $C$ [Fig. 3(a)]. The wave packet tunneling events for the chirped case are located at points $A^{\prime}$ and $B^{\prime}$, as shown in Fig. 3(b).

Figures 3(c) and 3(d) depict the electron recollision energy map. Each peak in the energy map denotes a recollision event. For the chirped case [Fig. 3(d)], there are two major emission events taking place. Event $A^{\prime}$ (not shown) is located at about -0.2 optical cycles (OC) in the lowerharmonic frequency side while the most prominent burst, event $B^{\prime}$, is situated approximately at 0.6 OC. Likewise, for the chirp-free case, there are three major rescattering events (labeled $A, B$, and $C$ ). The dark regions (in gray scale) as seen in Figs. 3(c) and 3(d) indicate a significantly larger number of trajectories than those of white regions. Recolli-
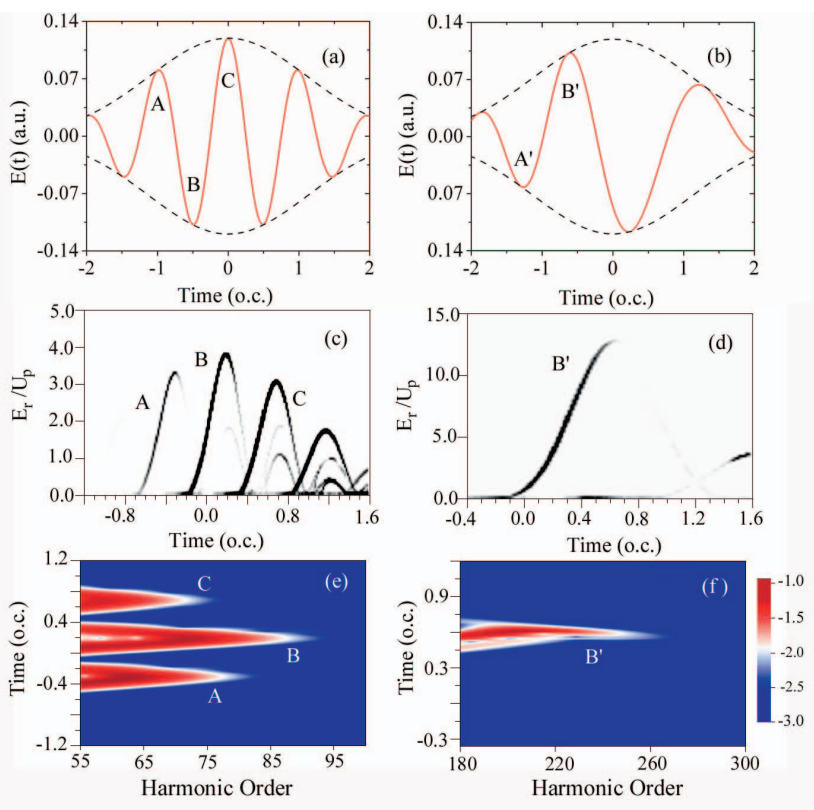

FIG. 3. (Color online). Chirp-free results are displayed in the left column, and chirped results in the right column $(\beta=6.25 \mathrm{rad}$, $\tau=200$ a.u.). (a), (b) Driving laser pulse and envelope; (c), (d) classical returning energy maps. Gray scale from maximum (black) to $1 / 10$ of maximum. (e), (f) Wavelet time-frequency profile $\left(\log _{10}\right.$ scale) of the HHG power spectra of hydrogen atoms driven by a few-cycle laser pulse. The chirp-free and chirped laser parameters are the same as those in Figs. 2(a) and 2(c), respectively. 

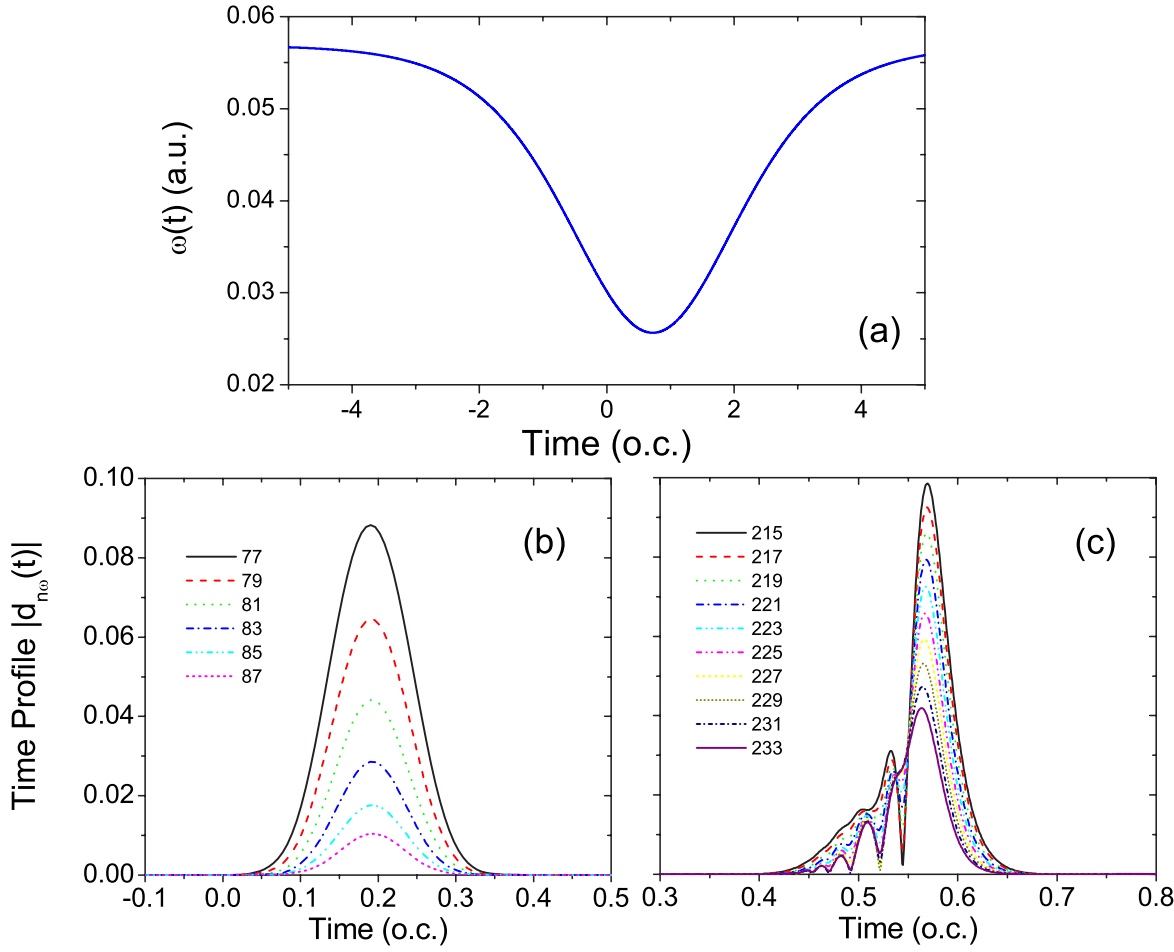

FIG. 4. (Color online) (a) Instantaneous frequency $\omega(t)$ of the chirped laser field. Laser parameters are the same as those in Fig. 2(c). Dipole time profiles of consecutive harmonics near the cutoff for the chirp-free (b) and chirped (c) laser pulses. sion of the electron with its parent ion is dominated by the "short trajectory" and can trigger the emission of an energetic attosecond pulse of (soft-X-ray) photons.

Figures 3(e) and 3(f) show the modulus of the quantum time-frequency profile corresponding to chirp-free and chirped laser pulses, respectively, obtained by the wavelet transform of the induced dipole acceleration $d_{A}(t)[7,13]$,

$$
A\left(t_{0}, \omega\right)=\int d_{A}(t) \sqrt{\omega} W\left(\omega\left(t-t_{0}\right)\right) d t \equiv d_{\omega}(t)
$$

For the chirped case, there is one major emission burst labeled $B^{\prime}$ in Fig. 3(f). Similarly, for the chirp-free case, there are three major emission bursts labeled $A, B$, and $C$ shown in Fig. 3(e). Events $B^{\prime}$ and $B$ are the most prominent bursts for the chirped and chirp-free laser pulses, respectively. Each burst emission time remarkably corresponds to the classical recollision time shown, in the corresponding returning energy-map figure [Figs. 3(c) and 3(d)]. This indicates that the HHG emissions are generated by a sequence of recollisions of the electron wave packet with the ion core $[20,27,30]$. Note that the maximum returning energy shown in Fig. 3(d) ( $B^{\prime}$ event) is considerably higher than that of the chirp-free case, shown in Fig. 3(c). As a result, the maximum photon energy of the cutoff harmonics ( $B^{\prime}$ event) of Fig. 3(f) is extended for at least 150 harmonic orders, compared with the chirp-free case [Fig. 3(e)].

A plausible explanation regarding the cutoff extension mechanism has to do with the excess energy acquired by the electron in the laser field. As the chirping becomes more pronounced close to the center of the laser pulse, the instantaneous frequency becomes increasingly smaller until reaching a minimum value. For the second half of the laser pulse, the instantaneous frequency $\omega(t)[\mathrm{Eq} .(4)]$ increases again. Since the pondermotive energy $U_{p}$ acquired by the electron is inversely proportional to the instantaneous frequency $\left[U_{p} \propto F / \omega^{2}(t)\right][$ Fig. 4(a)], the energy attained by the electron will achieve the maximum value near the minimum $\omega(t)$. In this manner, the electron has substantial more returning energy at the recollision event with the core, leading to the observed extension of the cutoff.

We accentuate that the present paper is a purely singleatom response study. In this manner, the treatment of the propagation effects caused by the medium is beyond the scope of the present investigation. We note that the commonly used cutoff law $I_{p}+3.17 U_{p}$, based on the single-atom response, is not strongly affected by the propagation effects. Furthermore, our few-cycle excitation study suggests that the short trajectory is the dominant contributor to the HHG and cutoff extension. It has been shown theoretically [28] and experimentally [29] that three-dimensional propagation effects favor contributions from the short trajectories. In this manner, each half cycle of the laser field results into a single dominant contribution to the high harmonics.

Finally, we note that the pulse duration of the attosecond burst produced by chirped pulses is substantially shorter than that of the chirp-free pulses. To substantiate such a fact, the dipole time profiles of the harmonics near the cutoff, obtained from the wavelet time-frequency analysis, are presented. We see that each consecutive harmonic is synchronized and the attosecond pulse width is reduced from $0.188 \pm 0.002$ OC for the chirp-free case [Fig. 4(b)] to $0.0404 \pm 0.002$ OC (108 as) for the chirped pulse laser [Fig. 4(c)]. Detailed discussion of the control and generation of attosecond pulses by few-cycle chirp-free laser fields was presented in [14]. We note that, in a different but related approach [31], it has been recently proposed that by chirping 
and optimizing the excitation laser pulse phase, single attosecond pulses may also be synthesized. Our goal in this paper is, however, quite different, and is focused on the optimization of the chirping parameter to achieve the HHG cutoff extension, leading to the possibility of the generation of attosecond pulses in the considerably higher-frequency regime.

In conclusion, we have presented a fully $a b$ initio quantum investigation and classical trajectory simulation for the exploration of the HHG cutoff extension phenomenon controlled by a few-cycle chirped laser pulse. It is shown that quantum and classical results provide complementary and consistent information regarding the mechanisms for the HHG cutoff extension. Furthermore, the time duration of the emitted attosecond bursts produced by the chirped laser pulse is significantly reduced from that of the chirp-free laser pulses.

This work was supported in part by the Chemical Sciences, Geosciences and Biosciences Division of the Office of Basic Energy Sciences, Office of Science, U.S. Department of Energy, and by NSF.
[1] A. Baltuška et al., Nature (London) 421, 611 (2003).

[2] Th. Udem, R. Holzwarth, and T. W. Hänsch, Nature (London) 416, 233 (2002).

[3] M. Hentschel et al., Nature (London) 414, 509 (2001).

[4] See, for example, I. P. Christov, M. M. Murnane, and H. C. Kapteyn, Phys. Rev. Lett. 78, 1251 (1997).

[5] Z. Chang, Phys. Rev. A 70, 043802 (2004).

[6] M. C. Stowe, F. C. Cruz, A. Marian, and J. Ye, Phys. Rev. Lett. 96, 153001 (2006).

[7] X. Chu and Shih-I Chu, Phys. Rev. A 64, 021403(R) (2001).

[8] R. A. Bartels, M. M. Murnane, H. C. Kapteyn, I. Christov, and H. Rabitz, Phys. Rev. A 70, 043404 (2004).

[9] See, for example, D. G. Lee, J. H. Kim, K. H. Hong, and C. H. Nam, Phys. Rev. Lett. 87, 243902 (2001).

[10] J. Lambert, M. W. Noel, and T. F. Gallagher, Phys. Rev. A 66, 053413 (2002).

[11] G. L. Yudin, A. D. Bandrauk, and P. B. Corkum, Phys. Rev. Lett. 96, 063002 (2006).

[12] X. M. Tong and Shih-I Chu, Chem. Phys. 217, 119 (1997).

[13] X. M. Tong and Shih-I Chu, Phys. Rev. A 61, 021802(R) (2000).

[14] J. J. Carrera, X. M. Tong, and Shih-I Chu, Phys. Rev. A 74, 023404 (2006).

[15] F. H. M. Faisal, Theory of Multiphon Processes (Plenum Press, New York, 1987), p. 9.

[16] S. T. Cundiff and J. Ye, Rev. Mod. Phys. 75, 325 (2003).

[17] C. W. S. Conover, M. C. Doogue, and F. J. Struwe, Phys. Rev.
A 65, 033414 (2002).

[18] See, for example, M. R. Hermann, and J. A. Fleck, Phys. Rev. A 38, 6000 (1988).

[19] X. M. Tong and Shih-I Chu, Phys. Rev. A 61, 031401(R) (2000).

[20] X. M. Tong and Shih-I Chu, Phys. Rev. A 64, 013417 (2001).

[21] X. Chu and Shih-I Chu, Phys. Rev. A 63, 023411 (2001); 64, 063404 (2001).

[22] X. Chu and Shih-I Chu, Phys. Rev. A 70, 061402(R) (2004).

[23] X. Guan, X. M. Tong, and Shih-I Chu, Phys. Rev. A 73, 023403 (2006).

[24] G. Yao and Shih-I Chu, Chem. Phys. Lett. 204, 381 (1993).

[25] V. Tosa, H. T. Kim, I. J. Kim, and C. H. Nam, Phys. Rev. A 71, 063808 (2005).

[26] P. B. Corkum, Phys. Rev. Lett. 71, 1994 (1993).

[27] M. Protopapas, D. G. Lappas, C. H. Keitel, and P. L. Knight, Phys. Rev. A 53, R2933 (1996).

[28] V. S. Yakovlev and A. Scrinzi, Phys. Rev. Lett. 91, 153901 (2003).

[29] L. C. Dinu, H. G. Muller, S. Kazamias, G. Mullot, F. Augé, Ph. Balcou, P. M. Paul, M. Kovačev, P. Breger, and P. Agostini, Phys. Rev. Lett. 91, 063901 (2003).

[30] J. J. Carrera, Shih-I Chu, and X. M. Tong, Phys. Rev. A 71, 063813 (2005).

[31] A. B. Yedder, C. Le Bris, O. Atabek, S. Chelkowski, and A. D. Bandrauk, Phys. Rev. A 69, 041802(R) (2004). 